\title{
Abstract: Semantic Technology at The New York Times: Lessons Learned and Future Directions
}

\author{
Evan Sandhaus
}

New York Times, US

\begin{abstract}
At last year's International Semantic Web Conference, The New York Times Company announced the release of our Linked Open Data Platform available at http://data.nytimes.com. In the subsequent year, we have continued our efforts in this space and learned many valuable lessons. In our remarks, we will review these lessons; demonstrate innovative prototypes built on our linked data; explore the future of RDF and RDFa in the News Industry and announce an exciting new milestone in our Linked Data efforts.
\end{abstract}

